\title{
Recent aspects on possible interrelation between precursory electric signals and anomalous bioeffects
}

\author{
E. Dologlou \\ Solid State Section, Department of Physics, University of Athens, Panepistimiopolis, Zografos 15784 Athens, Greece
}

Received: 22 June 2010 - Revised: 2 August 2010 - Accepted: 3 September 2010 - Published: 20 September 2010

\begin{abstract}
A possible geophysical mechanism based on the conception of criticality and on new aspects of biological effects caused by electromagnetic fields is discussed as a stimulus to the unusual animal behavior prior to large earthquakes. This mechanism is related to transient electric signals of low frequency and intensity, which are emitted from the pre-focal area several days before the impending earthquake and fulfill all the conditions set by a recent bioeffect model.
\end{abstract}

\section{Introduction}

One of the most challenging targets of seismology is the detection of precursors implying the forthcoming of large earthquakes. Among various physical phenomena observed prior to earthquakes is the unusual animal behavior which got controversial arguments from the scientific community about its credibility.

Since ancient times people believed that animals can sense impending earthquakes and instinctively try to escape and rescue their lives. The most impressive case is the successful prediction of Haicheng earthquake in 1975 (Scholz, 1977; Qidong et al., 1981), when as the main shock was approaching a growing number of different animal species (chicken, fish, dogs, snakes, etc.) exhibited an anomalous behavior systematically reported at various communes in the pre focal area.

Living organisms have the ability, known as homeostasis, to regulate their inner environment to ensure its stability in response to fluctuations in the outside environment. An organism may be a conformer or a regulator. Conformers allow the environment to determine any given life parameter

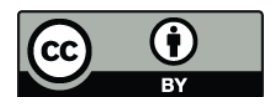

Correspondence to: E. Dologlou (edologl@phys.uoa.gr) like the temperature (i.e., endorthermic animals such as reptiles) while regulators try to maintain the parameter to a constant level over wide ambient environmental variations (i.e., exothermic animals such as mammals). Homeostatic control mechanisms have at least three main components; The receptor which is the sensing component that monitors and responds to the changes of the environment. The control center (e.g., the brain) that receives the information sent by the receptor and determines an appropriate response to the stimulus and the effector (e.g., muscles or other organs) which after receiving the signal from the control center, tries to correct the deviation by either enhancing it with positive feedback or depressing it with negative feedback.

Some animals are much more capable than humans of perceiving certain kinds of precursory phenomena. Different geophysical stimuli have been suggested to explain this abnormal behavior. Animals can sense and respond to seismic or acoustic waves at low frequency $(<50 \mathrm{~Hz})$, such as the small amplitude seismic P-waves (which travelling faster than the destructive shear S-waves outrun them by some seconds and arrive first on the earth's surface) although this response can be considered rather as an "early warning" than a true precursor (Kirschvink, 2000).

Among the various senses there is also the electric field sense which is the biological ability of an animal to perceive electrical impulses (Pettigrew, 1999). It is found (so far) only in aquatic animals: fishes from the Class Chondrichthyes and also some other fishes: from the family Mormyridae, from the order Gymnotiformes and in many catfishes. It is also found in the bill of Platypus, an aquatic mammal and possibly in toads since some external factors, as the electric field variation for example, may be detected in more hidden way - without any specialized sense.

Electric field sense can be characterized as an active or passive electroreception. In active electroreception the animal senses its surrounding environment by generating electric fields and detecting distortions in these fields using

Published by Copernicus Publications on behalf of the European Geosciences Union. 
electroreceptor organs which are sensitive to high frequency $(20-20000 \mathrm{~Hz})$ stimuli. Passive electroreception however, relies upon ampullary receptors which are sensitive to low frequency stimuli (below $50 \mathrm{~Hz}$ ).

In addition, fluctuations in the concentration of the radioactive gas Radon in ground water and pulsed electromagnetic waves (Ikeya et al., 2000), may trigger animals while certain fish are sensitive to very small electric field changes as $10^{-5} \mathrm{~V} / \mathrm{m}$ (Buskirk et al., 1981; Chu et al., 1996). Intense EM anomalies caused by seismic electromagnetic signals and unusual animal behavior have been reported before the earthquakes of Kobe-Japan in 1995 and IzmitTurkey in 1999 (Ikeya et al., 2000). Such anomalies reproduced in the laboratory using pulsed EM waves were linked with the abnormal animal behavior. Reports on the orientation of fish and silkworm before the Kobe earthquake suggested that their strange behavior might be attributed to the electric and not the magnetic field of pre-seismic EM waves (Ikeya et al., 2000).

Scientists have repeatedly observed (Liboff, 1985; Vaughan and Weaver, 1996; Weaver et al., 2000; Panagopoulos et al., 2000) that weak electromagnetic fields can produce biological effects. The last three decades changes of the earth's electrotelluric field, the so called Seismic Electric Signals (SES), have been detected prior to large earthquakes in Greece (Varotsos and Alexopoulos, 1984a, b; Varotsos et al., 1986, 1993a). An interconnection between those SES signals with bioeffects has been attempted by Filippetis (2009). Very recently, an article (Grant and Halliday, 2010) reporting anomalous animal behavior prior to the destructive earthquake of L'Aquilla - Italy in 2009, draw a lot of interest and publicity. It states that toads showed a dramatic change in their behavior 5 days before the earthquake and resumed their normal life some days after the event. Similar behavior of toads abandoning their usual living places (marshes, ponds) and filling the streets was also reported two days before the deadly earthquake of $M_{\mathrm{w}} 7.9$ in May 2008 in Sichuan - China (http://yeinjee.com/2008/ toad-migration-in-jiangsu-china/).

According to Grant and Halliday (2010) the geophysical stimuli responsible for this anomalous behavior of toads were VLF perturbations in the ionosphere five days before the L'Aquilla earthquake.

In this paper we will examine possible alternative geophysical mechanisms, based on the conception of criticality (i.e. SES activity) and on new aspects of the biological effects of the electromagnetic fields (Panagopoulos et al., 2000), which could provoke this highly unusual behavior of toads and consequently of other animal species before large earthquakes. We will present in brief the bioeffect model of Panagopoulos et al. (2000) and the physical meaning and properties of the preseismic electric SES signals.

\section{Bioeffects caused by electromagnetic signals: a plausible mechanism}

Various mechanisms have been proposed to explain unusual biological reactions to electromagnetic EM anomalies. A recent mechanism (Panagopoulos et al., 2000) suggests that low frequency electric fields can trigger bioeffects. According to this model, on both sides of every cell membrane there are free ions (mainly $\mathrm{K}^{+}, \mathrm{Na}^{+}, \mathrm{Cl}^{-}, \mathrm{Ca}^{2+}$ ) which create the strong electric field. An external alternating electric field will cause on every free ion a forced vibration. The oscillating ions, representing a periodical displacement of electric charge, are able to exert forces on every fixed charge of the membrane, like the charges on sensors of voltage-gated channels and hence to upset the membrane's electrochemical balance. The fixed charges on the gates of the ion transport cannot considerably vibrate due to the much greater mass of these structures. Voltage-gated channels are ions $\mathrm{K}^{+}, \mathrm{Na}^{+}$leak channels and their open or closed status is determined from electrostatic interaction between the channels' voltage sensors and the transmembrane voltage. At a point at which the electrostatic force on the channel voltage sensors exceeds a certain value the channel switches between the open and closed states. Changes of about $30 \mathrm{mV}$ in membrane potential are able to gate the channels. We must note that there are several kinds of gates/channels for the transport of ions through the cell membrane and voltagegated channels are only one group of those. Summarizing the above bioeffect model the following conclusion of great importance for the present work emerges: external electric field of low frequencies (below $1000 \mathrm{~Hz}$ ) can be bioactive even at very low intensities of some $\mathrm{V} / \mathrm{m}$. As the frequency of the field increases more than $10^{3} \mathrm{~Hz}$ the minimum intensity able to cause biological effects increases linearly with frequency.

\section{Seismic electric signals: theory and observations}

Let us now examine how the area around the epicenter of the impending earthquake is organized and what are the pre-seismic SES signals. Pop science strongly supports the concept that earthquakes belong to critical phenomena and power law processes prevail in the pre-seismic volume (Bowman et al., 1998; Sornette, 1989). When the accumulated tectonic stress in the pre focal area reaches a critical value, according to a model proposed by the Varotsos and co-workers, a transient electric current of low frequency $(\sim 1 \mathrm{~Hz})$ is emitted, known as SES signal (Varotsos and Alexopoulos, 1984a, b). Since early eighties, SES signals have been detected prior to large earthquakes in Greece. A brief description of the physical mechanism of the SES generation is as follows: The solid materials in the focal area of an impending earthquake contain intrinsic defects (e.g. vacancies - Varotsos and Alexopoulos, 
1979, 1984c; Varotsos, 2007, interstitials - Varotsos and Alexopoulos, 1978a; Varotsos, 1976) as well as extrinsic defects when aliovalent impurities are present (Varotsos and Alexopoulos, 1977, 1978b). Before an earthquake, the tectonic stress (pressure) increases gradually in the candidate focal area and causes changes in different physical properties as conductivity (Varotsos and Mourikis, 1974; Varotsos, 1980a), dielectric constant (Varotsos, 1978, 1980b) etc. Furthermore, the relaxation time of electric dipoles, formed in an ionic solid between aliovalent impurities (Varotsos and Miliotis, 1974) and vacancies created for electrical neutrality, (Kostopoulos et al., 1975) and depending on both temperature and pressure, is also affected. This may decrease (Varotsos and Alexopoulos, 1980b) upon increasing the pressure. When the pressure reaches a critical value the dipoles of solids in the rocks in the pre-seismic volume, change orientation and a transient current, which constitutes the SES signal, is emitted. Some interesting characteristics of the SES signals (Varotsos and Alexopoulos, 1984a, b; Varotsos et al., 1993b) are: the lead time, $\Delta t$, which is the time difference between the SES detection and the earthquake occurrence and can vary from a few hours to some weeks; Recently, the introduction of a new time domain, termed natural time (Varotsos et al., 2002, 2003a, b, c, 2005; Abe et al., 2005), allowed the identification of the time of the impending earthquake with accuracy of the order of few days (Varotsos et al., 2006a, b). The epicentral area is estimated on the basis of the so called SES selectivity which states that a SES station can be sensitive to some specific seismic areas while remains inactive to some others even at closer distances (Varotsos et al., 1993b). The amplitude $E=\Delta V / L$ of the SES (where $\Delta V$ is the potential change measured by a dipole of two electrodes buried in the ground at a distance $L$ ) and the magnitude $M$ of the subsequent impending earthquake are interrelated by the experimental formula:

$\log E=\alpha M+b$

where $\alpha \simeq 0.3-0.4$ and $b$ is a site-dependent constant. Interestingly, Eq. (1) was found (Varotsos and Alexopoulos, $1984 a, b)$ to be of universal nature in the sense that is valid for all measuring sites and corresponding pre-seismic regions. According to Sornette et al. (1989) fracture processes exhibit critical behavior expressed by a power law relation with critical exponent values of 0.33 and 0.47 for 2 and 3 dimensions, respectively. The picture of similar critical exponents in fracture processes and in the experimental Eq. (1) probably implies that upon the emission of the SES the pre focal area enters into a critical stage. With the aid of a specific method, based on "natural time" analysis (Varotsos et al., 2006a, b) of the gradually evolving seismicity in the candidate focal area a better approximation of the critical point which signals the occurrence of the earthquake is achieved. The concept of "natural time" considers the order evolution of events (i.e. 1st, 2nd, etc.) instead of the conventional time evolution (Varotsos et al., 2004 and references therein).

During this preparatory critical stage, fracture processes exhibiting power-law scaling behaviour prevail in the preseismic area (Bak, 1997). Scaling behaviour (or scalefree behaviour) means that no characteristic scales dominate the dynamics of the underlying process and reveals the development of long range correlations that decay more slowly and extend over larger distances in time and space than the mechanisms of the underlying process would suggest (Barabási and Stanley, 1995; Bak, 1997). As a result other than SES precursory phenomena such as electromagnetic anomalies of higher frequencies (i.e. MHz, kHz) (Eftaxias et al., 2002; Kapiris et al., 2003) changes in the concentration of the radioactive gas Radon (Omori et al., 2007; Plastino et al., 2010), increase of the specific resistivity of rocks, changes in the water level in wells as well anomalous animal behaviour appear in the pre focal area.

\section{Precursory SES signals and anomalous bioeffects}

We remind that the model of Panagopoulos et al. (2000) states that biological effects can be produced by low frequency and very low intensity electric fields. These are features that also characterize the SES signal which has a very low frequency $(<1 \mathrm{~Hz})$ and an amplitude of the order of several $\mathrm{mV} / \mathrm{km}$ at epicentral distances of $100 \mathrm{~km}$ (closer to the epicentre and for large earthquakes $(M>6)$ the SES amplitude will be significantly larger). Besides the SES activity that precedes the earthquake by several days to few weeks additional electric pulses of short duration appear few seconds before the main shock at SES sensitive sites with an amplitude of the order of $\mathrm{mV} / \mathrm{m}$ (Varotsos and Alexopoulos, 1986).

Considering the above bioeffect model we alternatively suggest that probably mechanisms related to SES activity close to the future epicentre might have stimulated the highly anomalous behaviour of the toads (Grant and Halliday, 2010) before L'Aquilla earthquake. Reasonably, it is more probable that aquatic larvae of toads would sense small variation of electric field than do the adult, land-dwelling animals because in water, electric fields cause the electric currents while the intensity of such currents in the air is very low. However, it is possible that the damp skin of the toad, in contact with the burrow, may react to the small changes of electric field. These changes, generated in seismic-sensitive area (or in the area where pre-earthquake processes occur), may be transmitted to the soil and to the toads' burrows, even in the urban area. The proposed by Grant and Halliday (2010) geophysical stimulus of VLF/LF ionospheric perturbations is a long space scale anomaly 
that should affect the behaviour of animals living in a considerably much larger area, even in another than Italy country, and not only close to the epicentre where the SES source is.

\section{Conclusions}

Here, we suggest that the reported anomalous behaviour of toads few days before the L'Aquilla earthquake in 2009 (Grant and Halliday, 2010), might rather be stimulated by processes related to SES activities developed during the critical stage in the pre focal area than to VLF/LF ionospheric perturbations. This argument is supported by the following two key-points: first, the SES signals fulfil all the conditions set by the bioeffect model of Panagopoulos et al. (2000), i.e. an electric field of low frequency and low intensity, and second, SES are emitted from the small scale pre-seismic area where the highly strange toads behaviour was observed.

Edited by: M. E. Contadakis

Reviewed by: two anonymous referees

\section{References}

Abe, S., Sarlis, N. V., Skordas, E. S., Tanaka, H. K., and Varotsos, P.: Origin of the usefulness of the natural time representation of complex time series, Phys. Rev. Lett., 94, 170601, 4 pp., 2005

Bak, P.: How nature works, Oxford, Oxford UP, 1997.

Barabasi, A. L. and Stanley, H. E.: Fractal Concepts in Surface Growth, Cambridge University Press, Cambridge, 1995.

Bowman, D. D., Ouillon, G., Sammis, C. G., Sornette, A., and Sornette, D.: An observational test of the critical earthquake concept, J. Geophys. Res.-Sol. Ea., 103, 24359-24372, 1998.

Bushirk, R. E., Frohlich, C., and Latha, G. V.: Unusual animal behaviour before earthquakes: A review of possible sensory mechanisms: Rev. Geophys. Space Phys., 19, 247-270, 1981.

Chu, J., Gui, X., Dai, J., Marone, C., Spiegelman, M., Seeber, L., and Armbruster, J.: Geoelectric signals in China and the earthquake generation process, J. Geophys. Res., 101, 1386913882, 1996.

Eftaxias, K., Kapiris, P., Dologlou, E., Kopanas, J., Bogris, N., Antonopoulos, G., Peratzakis, A., and Hadjicontis, V. : EM anomalies before the Kozani earthquake: A study of their behavior through laboratory experiments, Geophys. Res. Lett., 29, 1-69, 2002.

Filippetis, A. L.: Electromagnetic signals before rupture and their possible interconnection with bioeffects, Mod. Phys. Lett. B, 23, 1431-1436, 2009.

Grant, R. A. and Halliday, T.: Predicting the unpredictable; evidence of pre- seismic anticipatory behaviour in common toad, J. Zool., 281(4), 9 pp., ISSN:0952-8369, 2010.

Ikeya, M., Yamanaka, C., Mattsuda, T., et al.: Electromagnetic pulses generated by compression of granitic rocks and animal behaviour, Episodes, 23, 262-265, 2000.
Kapiris, P. G., Eftaxias, K. A., Nomikos, K. D., Polygiannakis, J., Dologlou, E., Balasis, G. T., Bogris, N. G., Peratzakis, A. S., and Hadjicontis, V. E.: Evolving towards a critical point: A possible electromagnetic way in which the critical regime is reached as the rupture approaches, Nonlin. Processes Geophys., 10, 511524, doi:10.5194/npg-10-511-2003, 2003.

Kirschvink J.: Earthquake Prediction by Animals: Evolution and Sensory Perception, B. Seismol. Soc. Am., 90, 312-323, 2000.

Kostopoulos, D., Varotsos, P., and Mourikis, S.: Conductivity of crystalline NaI, Can. J. Phys., 53, 1318-1320, 1975.

Liboff, A.: Interactions Between Electromagnetic Fields and Cells, Plenum, London, 1985.

Omori, Y., Yasuoka, Y., Nagahama, H., Kawada, Y., Ishikawa, T., Tokonami, S., and Shinogi, M.: Anomalous radon emanation linked to preseismic electromagnetic phenomena, Nat. Hazards Earth Syst. Sci., 7, 629-635, doi:10.5194/nhess-7-629-2007, 2007.

Panagopoulos, D. J., Messini, N., Karabarbounis, A., Filippetis, A. L., and Margaritis, L. H.: A mechanism for Action of Oscillating Electric Fields on Cells, Biochem. Biophys. Res. Commun., 272, 634-640, 2000.

Pettigrew, J. D: Electroreception in Monotremes, J. Exp. Biol., 202, 1447-1454, 1999.

Plastino, W., Povinec, P., De Luca, G., Doglioni, C., Nisi, S., Ioannucci, L., Balata, M., Laubenstein, M., Bella, F., and Coccia, E.: Uranium groundwater anomalies and L'Aquila earthquake, 6th April 2009 (Italy), J. Environ. Radioactiv., 101, 45-50, 2010.

Qidong, D. and $\mathrm{Pu}$, J.: A preliminary analysis of reported changes in ground water and anomalous animal behaviour before the 4 February 1975 Haicheng earthquake Earthquake prediction: an international review, American Geophysical Union, Washington, DC, 1981.

Scholz, C. H.: A physical interpretation of the Haicheng earthquake prediction, Nature, 267, 121-124, 1977.

Sornette, D., Lagier, M., Roux, S., and Hansen, A.: Critical piezoelectricity in percolation, J. Physiology-Paris, 50, 22012216, 1989.

Varotsos, P.: Comments on the formation entropy of a Frenkel defect in $\mathrm{BaF}_{2}$ and $\mathrm{CaF}_{2}$, Phys. Rev. B, 13, p. 938, 1976.

Varotsos, P. A.: An estimate of the pressure dependence of the dielectric constant in alkali halides, Phys. Status Solidi B, 90, 339-343, 1978.

Varotsos, P.: On the temperature variation of the bulk modulus of mixed alkali halides, Phys. Status Solidi B, 99, K93-K96, 1980a.

Varotsos, P.: Determination of the dielectric constant of alkali halide mixed crystals, Phys. Status Solidi B, 100, K133-K138, 1980 b.

Varotsos, P.: Comparison of models that interconnect point defect parameters, J. Appl. Phys., 101, 123503-123503-8, doi:10.1063/1.2745359, 2007.

Varotsos, P. and Mourikis, S.: Difference in conductivity between LiD and LiH crystals, Phys. Rev. B, 10, 5220-5224, 1974.

Varotsos, P. and Miliotis, D.: New aspects on the dielectric properties of the alkali halides with divalent impurities, J. Phys. Chem. Solids, 35, 927-930, 1974.

Varotsos, P. and Alexopoulos, K.: Estimation of the migration enthalpy and entropy for cation vacancy motion in alkali halides with the NaCl-type structure, Phys. Rev. B, 15, 2348-2351, 1977.

Varotsos, P. and Alexopoulos, K.: The curvature in conductivity 
plots of silver halides as a consequence of anharmonicity, J. Phys. Chem. Solids, 39, 759-761, 1978a.

Varotsos, P. and Alexopoulos, K.: Calculation of the migration volume of vacancies in ionic solids from macroscopic parameters, Phys. Status Solidi A, 47, K133-K136,1978b.

Varotsos, P. and Alexopoulos, K.: On the possibility of the enthalpy of a Schottky defect decreasing with increasing temperature, J. Phys. Solid State, 12, L761-L764, 1979.

Varotsos, P. and Alexopoulos, K. : Physical properties of the variations of the electric field of the earth preceding earthquakes, I, Tectonophysics, 110, 73-98, 1984a.

Varotsos, P. and Alexopoulos, K.: Physical properties of the variations of the electric field of the earth preceding earthquakes, II. Determination of epicentre and magnitude, Tectonophysics, 110, 99-125, 1984b.

Varotsos, P. and Alexopoulos, K.: Connection between the formation volume and formation Gibbs energy in noble gas solids, Phys. Rev. B, 30, 7305-7306, 1984c.

Varotsos, P. and Alexopoulos, K.: Thermodynamics of Point Defects and their Relation with the Bulk Properties, North Holland, Amsterdam, 1986.

Varotsos, P., Alexopoulos, K., and Lazaridou M.: Latest aspects of earthquake earthquake prediction in Greece based on Seismic Electric Signals II, Tectonophysics, 224, 1-37, 1993 b.

Varotsos, P., Alexopoulos, K., Lazaridou, M., and Nagao, T.: Earthquake predictions issued in Greece by Seismic Electric Signals since February 6. 1990, Tectonophysics, 224, 269-288, 1993a.

Varotsos, P. A., Sarlis, N. V., and Skordas, E. S.: Long-range correlations in the electric signals that precede rupture, Phys. Rev. E, 66, 011902, 7 pp., 2002.

Varotsos, P. A., Sarlis, N. V., and Skordas, E. S.: Long-range correlations in the electric signals that precede rupture: further investigations, Phys. Rev. E, 67, 021109, 13 pp., 2003a.
Varotsos, P., Sarlis, N., and Skordas, E.: Attempt to distinguish electric signals of a dichotomous nature, Phys. Rev. E., 68, 031106, 7 pp., 2003b.

Varotsos, P. A., Sarlis, N. V., and Skordas, E. S.: Electric fields that "arrive" before the time-derivative of the magnetic field prior to major earthquakes, Phys. Rev. Lett., 91, 148501, 4 pp., $2003 \mathrm{c}$.

Varotsos, P., Alexopoulos, K., Nomicos, K., and Lazaridou, M.: Earthquake prediction and electric signals, Nature, 322, p. 120 , 1986.

Varotsos, P. A., Sarlis, N. V., Skordas, E. S., and Lazaridou, M.: Natural entropy fluctuations discriminate similar looking electric signals emitted from systems of different dynamics, Phys. Rev. E, 71, 011110, 11 pp., 2005.

Varotsos, P. A., Sarlis, N. V., Skordas, E. S., and Lazaridou, M. S.: Entropy in the natural time-domain, Phys. Rev. E, 70, 011106, doi:10.1103/PhysRevE.70.011106, 2004.

Varotsos, P., Sarlis, N., Skordas, E., Tanaka, H., and Lazaridou, M.: Entropy of seismic electric signals: analysis in natural time under time reversal, Phys. Rev. E, 73, 031114, 8 pp., 2006 a.

Varotsos, P., Sarlis, N., Skordas, E., Tanaka, H., and Lazaridou, M.: Attempt to distinguish long-range temporal correlations from the statistics of the increments by natural time analysis, Phys. Rev. E, 74, 021123, 12 pp., 2006b.

Vaughan, T. E. and Weaver, J. C.: Energetic constraints on the creation of cell membrane pores by magnetic particles, Biophys. J., 71, 616-622, 1996.

Weaver, J. C., Vaughan, T. E., and Astumian, R. D.: Biological sensing of small field differences by magnetically sensitive chemical reactions, Nature, 405, 707-709, 2000. 